\title{
LE PORTUGAL ET LA FLANDRE DANS LE CADRE DE L'EXPANSION MARITIME DU PORTUGAL
}

\section{J. VERISSIMO SERRAO ${ }^{\circ}$}

1. Les relations entre le Portugal et la Flandre sont bien antérieures au XVième siècle. On trouve, en effet, des Flamands parmi les croisés qui aidèrent le roi Alphonse Henri, en l'an 1147 lors de la prise de Lisbonne. La princesse Thérèse, fille du même roi, s'est mariée en 1178 avec Philippe d'Alsace, comte de Flandre. Vers l'an 1200 son neveu Fernand, fils du roi Sanche, s'installa en pays flamand lors de son mariage avec Jeanne, duchesse de Flandre. A cette époque, on remarque aussi l'existence de plusieurs Flamands qui s' installèrent au Portugal pour aider au peuplement du royaume le plus occidental de l'Europe. Pour approfondir ces questions, nous avons recours aux ouvrages toujours actuels du Baron de Reifenberg, Coup d'oeil sur les relations qui ont été jadis entre la Belgique et le Portugal (Bruxelles, 1841) et d'Emile Vanden Bussche, Mémoire sur les relations qui existèrent autrefois entre les Flamands de Flandre - particulièrement ceux de Bruges - et les Portugais (Bruges, 1874).

En ce qui concerne les rapports économiques, nous savons qu'un bateau portugais est venu à Bruges en 1194, apportant une cargaison de bois, d'huile d'olive et de miel. D'autres produits en provenance du Portugal étaient vendus en Flandre, tels le vin, le sel et les fruits secs d'Algarve, tandis que les Portugais achetaient sur les marchés flamands des tissus, du froment et des objets en cuivre. Ce commerce est devenu important au cours du XIVième siècle, comme en témoigne un sauve-conduit du 15 janvier 1387 qui accordait des privilèges spéciaux "aux marchands et maîtres de navires des royaumes de Portugal et des Algarves". Les comtes de Flandre leur accordaient l'autorisation de vivre avec leurs familles et leurs serviteurs dans toutes les contrées du pays flamand pour y faire du commerce et du matelotage.

2. La ville de Bruges était l'entrepôt préféré des Portugais qui venaient en Flandre, étant donné le prestige qu'ils y avaient conquis tout au long de deux siècles. Ceci explique les lettres patentes de Jean, duc de Bourgogne, du 26 décembre 1411 , leur donnant permission pour la création d'une "nation" à Bruges. Cela veut dire, d'une corporation de marchands identique à beaucoup

`Université de Lisbonne 
d'autres qui existaient dans plusieurs ports de mer de l'Atlantique et de la Méditerranée. Le rôle du commerce avec le Portugal était reconnu par la cour bourguignone et aussi par les autorités municipales et les marchands de Flandre. Un document de cette époque rapporte "les grands proufitz et biens qui viennent à noz subjiez et à nostre dict pais communaument pour le bien proufit et multipliement d'icellui nostre pais et avancement de nos subgiez". C'est-à-dire que les Portugais jouissaient alors de toute liberté pour la navigation et le commerce dans toutes les contrées de la maison ducale de Bourgogne.

Cependant, ne croyons pas que le commerce luso-flamand se réduisait au port de Bruges, pour l'achat, de préférence, de tissus qu'au Portugal on nommait des "brujas". D'autres ports de Flandre faisaient aussi l'objet de la visite des hommes d'affaire portugais. Une liste de tissus et d'objets en métal arrivés à Lisbonne en 1411 rapporte les toponymes suivants : "peças dhipre" (Ypres), de "comua" ou de "cominas" (Comines), de "pepelingas" (Poperinghe), de "rooles" (Roulers), de "mosteirol" (Montreuil), de "balençinas (Valencienne) et de "mostreuilles" (Montvilliers). Outre ces tissus, le commerce Portugais comprenait des vases ("bacyos") de Flandre en fer, en étain, en cuivre et en plomben provenance de l'intérieurdel'Allemagne. Tout ceci prouve l'étendue du commerce luso-flamand qui se dirigeait par la suite à Lisbonne et à Porto, sans oublier les ports secondaires de Vila do Conde, de Setúbal et d'Algarve.

Ces rapports commerciaux ont conduit à d'autres sortes de rapports entre les deux communautés. N'oublions pas le rôle spirituel de l'Ordre de SaintDominique auprès des corporations maritimes et commerçantes. Ce fut ainsi que le 25 mars 1410 les Dominicains de Bruges ont accordé l'utilisation de la chapelle de la Sainte-Croix dans leur monastère, aux marchands Portugais qui demeuraient ou étaient seulement de passage dans cette ville. Une sorte de réciprocité s'établit ainsi entre les deux congrégations religieuses, ce qui fut à l'origine de la décision prise par les Dominicains de Lisbonne de fonder chez eux une chapelle nommée des Flamands ou des Bourguignons. Tous ceux qui nient l'influence des facteurs spirituels dans le cadre des affaires économiques trouveront ici un bon exemple, à la lumière du XVième siècle, pour en faire une bonne démonstration. C'est-à-dire que les marchands cherchent à s'enrichir sans que leurs ambitions soient incompatibles avec leurs croyances religieuses.

3. Nous arrivons ainsi à la deuxième décade du XVième siècle, autrement dit à la même époque où l'on place les débuts des découvertes maritimes des Portugais. On ne doute pas que le pays flamand offrit un considérable apport, 
en l'an 1415, à la prise de Ceuta par le roy Jean Ier du Portugal.C'est à Bruges que l'on a préparé quelques bateaux et une partie du matériel de guerre expédié par la suite à Lisbonne. Dans la flotte portugaise on trouve des chevaliers flamands parmi lesquels les deux frères Philippe et Martin Capella, natifs de Bruges, qui participèrent à cet exploit guerrier. Au temps de la découverte de l'île de Madère, vers l'année 1421, le duc Philippe confirmait les chartes ducales de 1411 permettant aux marchands, aux marins et à d'autres serviteurs du roi de Portugal de renforcer leur commerce avec les ports de Flandre. Donc, ces relations étaient favorables au début de 1426, lorsque le prince Pierre, troisième fils du roi portugais, vint à Bruges pour resserer les liens d'amitié entre son royaume et le duché de Bourgogne.

Entre le mois de janvier et le printemps 1426 le prince adressa une lettre à son frère aîné, le prince Edouard, document dont la valeur historique pour le Portugal et la Flandre est indéniable. Les historiens en parlent comme étant "la lettre de Bruges" puisque Pierre était venu dans cette ville pour y proposer le mariage de sa soeur Isabelle avec Philippe le Bon, duc de Bourgogne. Cette missive ayant été concluante, une ambassade fut envoyée au Portugal pour mettre au point l'alliance luso-bourguignonne. Parmi les délégués de Philippe on trouve le célèbre peintre Van Eyck, qui avait pour tâche de dessiner le portrait de la future duchesse. Selon les auteurs de son temps, Isabelle était une femme d'une rare beauté, sans doute issue de sa branche maternelle anglaise.

C'est un événement bien connu qu'Isabelle arriva au port de l'Ecluse le jour de Noël de l'an 1429 et qu'elle s'est rendue par la suite à Bruges où le 7 janvier suivant eût lieu la cérémonie du mariage ducal. La princesse s'était faite accompagner d'une escorte d'à peu près 2.000 personnes, étant reçue avec la plus grande splendeur. Parmi ces accompagnants Portugais, on comptait des nobles et des chevaliers, un grand nombre de dames de compagnie, sans oublier les religieux, les lettrés et les artistes qui allaient figurer dans sa maison seigneuriale. Je me pose la question de savoir si un certain nombre de peintres lusitaniens ne se sont pas rendus à cette époque-là en Flandre pour s'y perfectionner. Néanmoins il est bien prouvé qu'un certain João Vasques a rempli la charge de maître d'hotel de la duchesse et qu'il se maria à Bruges avec Catherine Van de Walle, habitant tous les deux à la rue d'Argent, dans une maison qui porte actuellement le numero 38.

Quelques centaines de Portugais se sont donc installés dans ces contrées de Flandre, tout en formant plusieurs noyaux d'immigrés qui ajoutèrent du sang portugais dans la société bourguignonne. Les fructueuses recherches du professeur Jacques Paviot dans le tissu historique bourgondo-flamand ont bien démontré le rôle de mes compatriotes au XVième siècle dans cette 
région de l'Europe. Et comment ne pas apprécier aussi la récente contribution de Madame dr. Monique Sommé au sujet des relations priviligiées que la duchesse Isabelle de Portugal a maintenues avec la ville de Bruges? Nous commençons donc à être bien renseignés sur la présence de tant de Portugais dans tous les échelons de la société flamande.

Je me permets encore d'attirer votre attention sur le contenu de la "lettre de Bruges" mentionnée ci-dessus du prince Pierre de Portugal, en tenant compte de la valeur de ce document pour l'histoire de Flandre. Pendant son séjour de quatre mois, le prince n'a pâ établir que quelques liens d'amitié ou de connaissance avec plusieurs personnes de Bruges. Ainsi, les conseillers du duc et les autorités municipales, sans oublier les hommes d'affaires et les consuls de la "nation" portugaise. Cepassage parl'un des centres commerciaux les plus renommés de l'Europe donna conscience à Pierre de perspectives nouvelles. Il a sans doute pressenti que le monde était en train de changer avec les mouvements de centralisation royaux et l'essor du capitalisme si évidents en Flandre et en Italie. Le prince Pierre de Portugal, tout en visitant Bruges, a bien compris le rôle des villes marchandes dans une Europe en quête d'un monde nouveau ouvert par l'entreprise maritime des Portugais. Permettez donc que je vous signale quelques données historiques sur l'expansion atlantique du Portugal.

4. A partir de la deuxième décade du Quatrocento, notre expansion maritime sous le patronnage de la couronne s'orienta dans trois directions. Tout d'abord les Portugais cherchèrent à s'emparer du Maroc, ce qui conduisit à la prise de Ceuta en 1415. Ce projet ne sera poursuivi que quarante années plus tard, lors de la conquête de Alcácer-Ceguer en 1458. La deuxième ligne de l'expansion avait pour but la découverte, suivie de la colonisation, des archipels de l'Atlantique, exception faite des Canaries qui restaient sous l'obédience de la Castille, soit Madère (1420-1421), les Açores (1427-1452), les îles du Cap Vert (1445) et celles de São Tomé et du Prince (1471). Enfin, la troisième ligne avait pour objectif de découvrir la côte occidentale de l'Afrique, à partir du Sahara jusqu'à l'Equateur. Autrement dit, les Portugais recherchaient le royaume du Prêtre Jean des Indes dont le souverain était un chrétien qui vivait entouré d'un monde d'infidèles.

Après plusieurs tentatives sans lendemain à partir de 1425 , neuf ans après, les Portugais ont pu dépasser le cap Bojador que l'imagination de l'époque considérait comme un endroit fermé à l'incursion des hommes. La côte africaine fut par la suite ouverte aux expéditions annuelles qui partaient de Lisbonne ou des ports du royaume d'Algarve. Ce fut ainsi que les Portugais ont découvert peu à peu le cap Blanc (1441), le Sénégal (1444), le Cap Vert 
(1445), la Sierra Leone (1450, le golfe de Guinée (1471), l'embouchûre du Zaïre ou Congo (1481), le littoral de l'Angola (1482) et le Cap de Bonne Espérance, atteint par Bartolomeu Dias en 1487. Cette entreprise se prolongea tout au long de 60 années, dans un effort maritime et financier sans pareil chez un petit peuple qui atteignait à peine 800 mille à un million d'habitants. Cependant, la côte occidentale de l'Afrique était ouverte à la connaissance des hommes et le visage du monde atlantique devint, après le voyage de Bartolomeu Dias, tout à fait différent.

Le rêve Portugais des "Indes" sera couronné lors du voyage de Vasco da Gama en 1497-1498, qui établit la première liaison directe entre Lisbonne et le Malabar. Par l'intermédiaire de l'Atlantique, l'Europe occidentale avait trouvé la seule route maritime directe pour atteindre l'Océan Indien. Après tant d'efforts et de sacrifices, les Portugais avaient accompli la tâche immense d'unir trois continents (l'Europe, l'Afrique et l'Asie) par la voie continue de deux mers (l'Atlantique et l'Océan Indien). Cen'était pas encore tout comme exploit de dimension universelle, car en 1500 une deuxième flotte envoyée aux Indes découvrit la côte du Brésil. Le succès de Cabral apporta à la couronne portugaise le Nouveau Monde baigné par l'Atlantique méridional. Ce fut ainsi qu'au début du XVlième siècle le Portugal était considéré en Europe comme la plus forte puissance dans les domaines politique, maritime et économique.

5. Nous sommes maintenant en mesure de comprendre le rôle de la Flandre dans le processus des découvertes portugaises. N'oublions pas que notre factorerie à Bruges formait un des entrepôts les plus actifs du commerce européen. Dans la première moitié du XVième siècle le nombre de bateaux en provenance de Lisbonne, de Porto, de Gaia, de Faro, de Tavira augmenta progressivement à Bruges et dans d'autres endroits du pays flamand. Le 2 novembre 1438 le duc Philippe accordait aux Portugais venus à l'Ecluse le privilège de vendre à bord de leurs navires l'écorce de lièges transportée, sans être obligés de décharger ce produit. Ainsi, pour bien comprendre l'état favorable de ces relations il suffit de dire que la duchesse Isabelle, elle même, était la propriétaire d'un bateau qui se rendait souvent au Portugal pour y faire du commerce sans en payer les dîmes ni d'autres droits.

Les découvertes maritimes ont changé beaucoup la variété des produits apportés en Flandre. Jusqu'à l'année 1450 s'est maintenu le commerce traditionel des excédents portugais : le vin, le sel, l'huile d'olive, les cuirs, les fruits secs et le miel. Au retour les bateaux en direction du Portugal rapportaient de la Flandre du poisson fumé, des chevaux, du froment et du 
blé, ainsi que des objets manufacturés et des armes de guerre. Mais à partir d'environ 1450-1460 de nouveaux produits provenant de l'economie atlantique arrivent. Ce fut d'abord le cas du miel et de la cire de Madère, auxquels s'ajoutèrent le vin et, en plus grosses quantités, le sucre qui devint la principale exportation vers la Flandre. Ce commerce était forcément compensé en espèces ou par échanges avec d'autres produits. Un fait à rappeler concerne la ville de Funchal dont le musée de la cathédrale conserve encore une dizaine de tableaux flamands (fin XVième siècle, début XVlième siècle) qui constituèrent la monnaie d'échange de certaines cargaisons de sucre vendu à Bruges et dans d'autres ports flamands.

En ce qui concerne l'archipel des Açores, il faut mentionner les plantes colorantes, tel le pastel et l'urzela, dont l'exportation est pouvée vers les ports septentrionaux de l'Atlantique, dont ceux de Flandre. Le succès de ces produits dépendait, en grande partie, de la loi de l'offre et de la demande des pays nordiques qui entretenaient des relations avec la factorerie de Bruges et, par la suite, avec celle à d'Anvers. Mais c'est un fait indéniable que des cargaisons de ces deux plantes furent préparées au marché de Lisbonne pour être vendues à ceux de Flandre. Dès 1470 on vendait aussi à Bruges la "malagueta" ou piment d'Afrique, ainsi que d'autres produits de la côte de Guinée. Sans oublier encore le sucre et le bois en provenance du Portugal et aussi les cordages, les tissus et d'autres outillages nautiques. Cependant, je ne vais pas poursuivre ces données historiques, car elles feront l'objet de la conférence du professeur Jorge Borges de Macedo.

On sait que la maison consulaire du Portugal exerçait déjà sa fonction à Bruges vers l'an 1445 et qu'elle jouissait dans cette ville d'une indéniable importance sociale. Les facteurs du roi portugais, comme Francisco Pessoa et João Brandão, tenaient ici un rang comparable à celui des ambassadeurs. En provenance des ports flamands et d'autres contrées du duché de Bourgogne, arrivaient au Portugal des livres, des meubles et des tapisseries qui ont garni les palais royaux, les bibliothèques des monastères et les hôtels particuliers de nombreux membres de la noblesse et de la bourgeoisie portugaise. Ces rapports intellectuels et artistiques, qui se sont développés par la suite lors du transfert de la factorerie à Anvers, apportèrent dans mon pays les bienfaits de votre culture et de votre manière de penser et de vivre. Sans parler de l'Université de Louvain qui a fort influencé les moines portugais de Saint Jérôme diplômés de cette fameuse institution.

6. Jusqu'à l'ensablement de sont port au XVIième siècle, la ville de Bruges joua ainsi un rôle très important pour le commerce Portugais, tout en étant la porte d'entrée de nos produits en direction de l'Empire et aussi de la mer 
Baltique. Ceci explique l'essor maritime d'Anvers qui remplaça Bruges comme le grand port de Flandre. La factorerie portugaise s'y installa dès l'an 1496, à la même époque où Lisbonne s'apprêtait à recevoir les nouvelles richesses orientales : les épices des Indes (le poivre, le gingembre, la noix, la canelle), ainsi que le fabuleux bois du Brésil, outre le sucre de l'île de São Tomé qui allait remplacer le sucre de Madère. Anvers ne fut pas seulement la seconde porte d'entrée en Flandre des produits en provenance de Lisbonne. Anvers fut aussi le lieu de contact des gens les plus renommés de cette époque : outre les banquiers et les bourgeois, on trouvait à Anvers des humanistes, des diplomates et des artistes, tous en quête de richesse et de gloire.

L'apogée de la factorerie anversoise est depuis longtemps connue, grâce aux travaux de J.A. Goris, Etude sur les colonies marchandes méridionales à Anvers, de 1488 à 1567 (Louvain, 1925) et plus récemment ceux de Renée Doehaerd, Etudes Anversoises (3 volumes, Paris, 1963) et du professeur de 1'Université de Pampelune, M. Valentín Vazquez de Prada, Lettres Marchandes d'Anvers (4 volumes, Paris, 1960-1966). Mais ne dépassons pas le cas historique de Bruges dans le cadre des relations luso-flamandes. D'ailleurs, beaucoup de questions devront être revues à la lumière de l'ouvrage du professeur Paviot, récemment paru.

7. Pour terminer, je voudrais encore dresser un court tableau concernant l'implantation de Flamands aux Açores. Nous savons qu'un document du régent Pierre de l'année 1439 témoigne du commencement de la colonisation des 7 îles déjà découvertes (sauf celles de Flores et du Corvo). Le royaume du Portugal pouvait alors compter 700 à 750.000 mille habitants et seules quelques dizaines de familles s'étaient fixés sur l'île de Madère pour y entreprendre la colonisation. Or, il n'y avait pas de colons métropolitains en nombre suffisant pour mener une tâche identique aux Açores.

Nous croyons donc que le Régent Pierre a fait une tentative auprès de sa soeur Isabelle pour attirer des gens de Flandre vers cet archipel. En ce temps-là, on trouve déjà quelques Flamands au Portugal, comme Martim Leme, "brujes de Brujas", qui se consacrait au commerce du liège. Il devait jouir d'une bonne réputation, car en 1450 il obtint du roi Alfonse $V$ des lettres de naturalisation. De même en ce qui concerne Gonçalo de Flandre qui faisait partie en 1437 de la maison de l'infant Fernand, également frère de la duchesse de Bourgogne. Dix ans plus tard un certain Jácome de Bruges vivait déjà au Portugal comme serviteur du prince Henri le Navigateur. Jácome se disait natif du comté de Flandre, avait une origine noble et possédait beaucoup de biens. D'après une charte du 2 mars 1450 il reçut la capitainerie 
de l'île de Terceira pour lui et ses descendants, ayant l'obligation de la faire peupler par d'autres personnes de foi catholique. Etant donné que nous ignorons tout au sujet de la tentative de colonisation des Açores en 1439, nous sommes donc en mesure de croire que Jácome de Bruges orienta la deuxième phase de la colonisation des Açores. Cependant, lorsque son travail était presque achévé, une grave crise politique éclata au Portugal, suite à la mort de l'ancien régent Pierre, tué lors de la rencontre d'Alfarrobeira. Ce drame survenu au sein de la famille royale produisit une vague d'émigration vers la Flandre. Les enfants du malheureux prince et quelques centaines de leurs serviteurs dûrent s'enfuir et trouvèrent refuge auprès de la duchesse Isabelle. Parmi eux on comptait des nobles, des chevaliers, des dames de cour, des religieux et des artistes qui se fixèrent pour toujours en pays flamand. Beaucoup d'entre eux se sont mariés en terre bourguignonne et il serait intéressant de connaître les branches seigneuriales qui les ont accueillis. Il y a une recherche à faire dans ce domaine qui pouvait conduire à des resultats surprenants, en tenant compte des savantes recherches de M. Paviot. Je veux seulement signaler le cas de Vasco de Lucena, né à Coimbra vers l'an 1430, par la suite étudiant à l'Université de Paris et qui jouissait de la protection de la duchesse Isabelle et de son mari. Lucena a traduit en français le De rebus gestis Alexandri Magni de Quinte-Curce, entre 1461 et 1467, sur l'ordre de Charles le Téméraire. On le considère aujourd'hui, par l'élégance de son style, comme l'un des meilleurs prosateurs en langue française de son temps.

En revenant encore au problème de la deuxième colonisation flamande aux Açores, il est certain que la crise politique de $1449-1450$ provoque un très long conflit entre le roi Alfonse $\mathrm{V}$ et la maison de Bourgogne. Je suis convaincu que tout cela aboutit à un considérable retard pour la fixation des colons aux Açores. Mais il est aussi prouvé qu'en 1466 la duchesse Isabelle obtint du roi de Portugal que Joost von Hurter, natif des environs de Bruges, puisse faire la colonisation de l'île de Faial. Aussi un Van der Hagen est parti avec sa famille vers les îles de Saint George et de la Graciosa. Ce fait coïncide avec le temoignage de Reifenberg, selon lequel en 1466 un groupe de deux mille Flamands commença le peuplement des "ilhas debaixo", c'est-à-dire celles de Graciosa, Saint-George et du Pico. 
Tout s'éclaircit donc, face au troisième moment de la colonisation des Açores par la voie flamande. Cela explique qu' aux îles nommées ci-dessus, on donne à la fin du XVième siècle le nom de "Ilhas Flamengas". Martin Behaim les mentionne dans son globe de Nuremberg et le médecin d'Augsburg, Jerôme Münzer aussi dans son Itinerario sur la péninsule Ibérique : "a do Faial, assim chamada porque havia lá muita madeira de faia, e a do Pico que são povoadas por 1500 pesoas dos dois sexos. O Faial tem um perimetro de 16 milhas e os habitantes falam todos o alemão da Flandres". Bref, ce problème n'est pas encore tout à fait résolu et plaise à Dieu que notre réunion puisse aboutir à de nouvelles recherches sur la colonisation flamande des Açores. 


\section{SAMENVATTING}

1. De relaties tussen Portugal en Vlaanderen gaan terug tot de 12de eeuw. Op dynastiek-politiek vlak speelde vooral het huwelijk van de Portugese koningsdochter Theresa met de Vlaamse graaf Filips van de Elzas een grote rol. Nog op het einde van de 12 de eeuw stelt men de eerste handelsactiviteiten van Portugezen in Brugge vast. Een eeuw later worden de handelsbetrekkingen echt belangrijk.

2. De Portugezen stichtten in de 15 de eeuw een natie in Brugge. Ook in andere Vlaamse steden waren zij als kooplui bedrijvig. Vanuit Vlaanderen werden goederen geëxporteerd naar Lissabon en Porto. Hiermee ging ook een spirituele factor gepaard, dankzij de gastvrijheid van de Dominicanen voor deze handelaars zowel in Brugge als in Lissabon.

3. Aan de Portugese ontdekkingstochten van de 15 de eeuw droegen de Vlamingen van meetaf aan een steentje bij. Door het huwelijk van de infante Isabella van Portugal met de Bourgondische hertog Filips de Goede werden de Portugees-Vlaamse betrekkingen nog intenser. Dit bracht de vestiging mee van een groot aantal Portugezen (hoflieden, handelaars, kunstenaars) in de Vlaamse steden.

4. Het Portugese avontuur van de ontdekkingen speelde zich af langs de Afrikaanse kusten en culmineerde op het einde van de 15de eeuw met de ontdekking van de doortocht naar Indië. Daardoor werd Portugal in Europa de sterkste macht op politiek, maritiem en economisch vlak.

5. Dankzij de ontdekkingen groeide de Portugese handesfactorij in Brugge uit tot een brandpunt van de Europese economie. Nieuwe producten, voornamelijk uit Madeira en de Afrikaanse kusten werden er op de markt gebracht.

6. Op het einde van de 15 de eeuw werd tengevolge van de achteruitgang van de Brugse haven, de Portugese factorij overgeplaatst naar Antwerpen. De toevloed van Oosterse producten kwam vooral de bloei van de Antwerpse haven ten goede.

7. Rest nog het probleem van de Vlaamse inbreng in de kolonisatie van de Azoren. Er was onvoldoende Portugese mankracht hiervoor, zodat via Isabella van Portugal een beroep gedaan werd op Vlamingen. De politieke crisis na de dood van de regent Pedro vertraagde wel deze kolonisatie en bracht ook een emigratie van Portugezen naar Vlaanderen teweeg. Nadien waren er nieuwe kolonisatiegolven van Vlamingen op de Azoren. Maar dit fenomeen moet nog verder worden onderzocht. 